\title{
Strawberry Gingivitis as the First Presenting Sign of Wegener's Granulomatosis: Report of A CASE
}

\author{
C. H. Siar ${ }^{1}$, K. B. Yeo ${ }^{2}$, K. Nakano ${ }^{3}$, H. Nagatsuka ${ }^{4}$, H. Tsujigiwa ${ }^{4}$, M. Tomida ${ }^{5}$, K. H. $\mathrm{Ng}^{6}$, \\ T. Kawakami ${ }^{3}$
${ }^{1}$ Department of Oral Pathology, Oral Medicine and Periodontology, Faculty of Dentistry, University of Malaya, Kuala Lumpur, Malaysia, ${ }^{2}$ Gleneagles Intan Medical Centre, Kuala Lumpur, Malaysia,
${ }^{3}$ Hard Tissue Pathology Unit, Matsumoto Dental University Graduate School of Oral Medicine, Shiojiri, Japan,
${ }^{4}$ Department of Oral Pathology and Medicine, Graduate School of Medicine, Dentistry and Pharmaceutical Sciences, Okayama University, Okayama, Japan,
${ }^{5}$ Department of Oral Physiology, Matsumoto Dental University School of Dentistry, Shiojiri, Japan,
${ }^{6}$ Formerly Unit of Stomatology, Cancer Research Centre, Institute for Medical Research, Jalan Pahang, Kuala Lumpur, Malaysia

\begin{abstract}
Wegener's granulomatosis is a rare multi-system disease characterized by the classic triad of necrotizing granulomas affecting the upper and lower respiratory tracts, disseminated vasculitis and glomerulonephritis. Oral lesions as a presenting feature are only encountered in $2 \%$ of these cases. Hyperplastic gingival lesions or strawberry gingivitis, is a characteristic sign of Wegener's granulomatosis. The latter consists of reddish-purple exophytic gingival swellings with petechial haemorrhages thus resembling strawberries. Recognition of this feature is of utmost importance for timely diagnosis and definitive management of this potentially fatal disease. A case of strawberry gingivitis as the first presenting sign of Wegener's granulomatosis affecting a 50-year-old Malay male is reported here. The differential diagnosis of red lesions that may present in the gingiva is discussed.
\end{abstract}

Key words: Wegener's granulomatosis, strawberry gingivitis, oral lesion, systemic disease,

\section{INTRODUCTION}

Wegener's granulomatosis is an uncommon multi-organ disease first categorized as a distinct syndrome by Friedreich Wegener in 1936 [1-3] The hallmarks of this potentially fatal disorder are necrotizing granulomatous inflammation involving the upper and lower respiratory tract, glomerulonephritis, and vasculitis [36]. Wegener's granulomatosis may occur as a limited or generalized disease. The limited form of Wegener's granulomatosis runs an indolent course whereas the disseminated disease has a rapid progressive course leading to life-threatening multi-organ failure [2, 710]. Of the 2 types of Wegener's granulomatosis, patients with the generalized disease are known to have shorter life expectancy than those presenting with the limited disease. Renal involvement is associated with a more severe outcome for these patients [5, 8]. Wegener's granulomatosis has an insidious onset and usually develops over a period of time with the mean period from onset of symptoms to diagnosis ranging from 4.7 to 15 months $[5,7,9]$. Without treatment it is invariably fatal and most patients do not survive more than a year after diagnosis [11-12]. Delay in the diagnosis of Wegener's granulomatosis is attributed mostly to the nonspecific presenting signs and symptoms associated with the early phase of the disease [3$4,8]$.

The most characteristic oral lesion is hyperplastic gingivitis, which is typically red to purple with many petechiae (strawberry gingivitis). These lesions may remain localized in the oral cavity for unusually long periods of time before multi-organ involvement occurs $[7,12]$. Therefore, timely recognition of this often overlooked oral finding can help to establish an early diagnosis of this disease $[4,8,11]$. Management with appropriate therapy produces a good response in most cases, with only occasional relapses.

\section{CASE REPORT}

A 50 year old male was referred to the Oral Medicine Clinic at the Department of Oral Pathology, Oral Medicine and Peridodontology, Faculty of Dentistry, University of Malaya for management of unusual gingival lesions. Accordingly, the patient first attended the Primary Dental Care Unit here for a routine dental check-up. During the course of intraoral examination, large lobulated purplish-red swellings were found affecting the labial gingival mucosa extending from the distal of the right maxillary first premolar to the distal of the left maxillary central incisor (Fig. 1A). The palatal gingival mucosa was uninvolved (Fig. 1B). The patient was unaware of the onset of these swellings, and there were no associated symptoms of pain or bleeding. No other lesions were found elsewhere in the oral cavity. Panoramic radiographs did not show any evidence of underlying bony involvement. He was afebrile at the time of examination and his medical history was otherwise unremarkable. 
Histopathological Findings

A decision was made to perform an incisional biopsy on the gingival growth under local anaesthesia. The specimen obtained was submitted for routine processing followed by staining with haematoxylin-eosin, Periodic acid Schiff, Grocott methenamine silver and Ziehl-Neelsen.
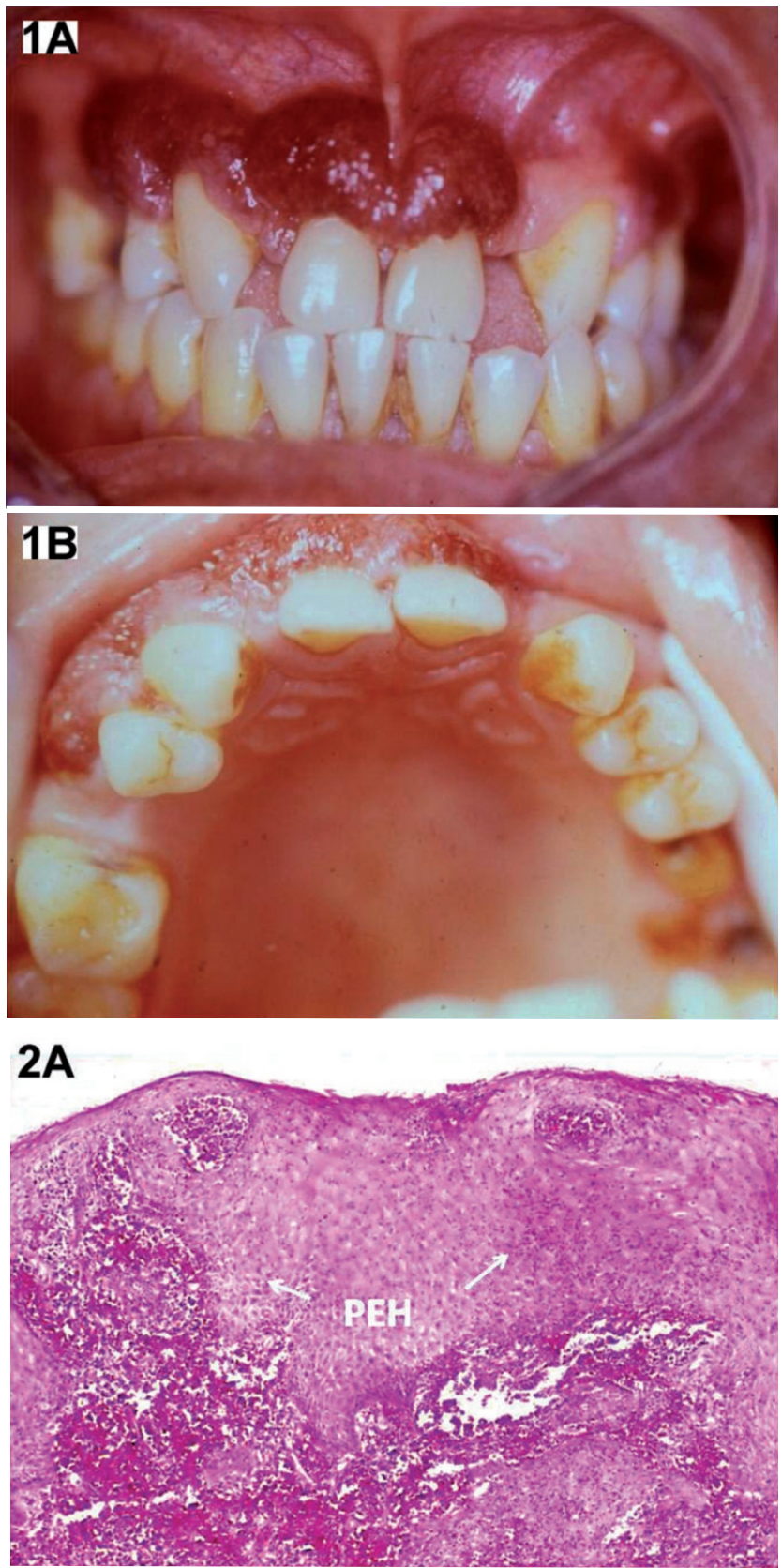

2B

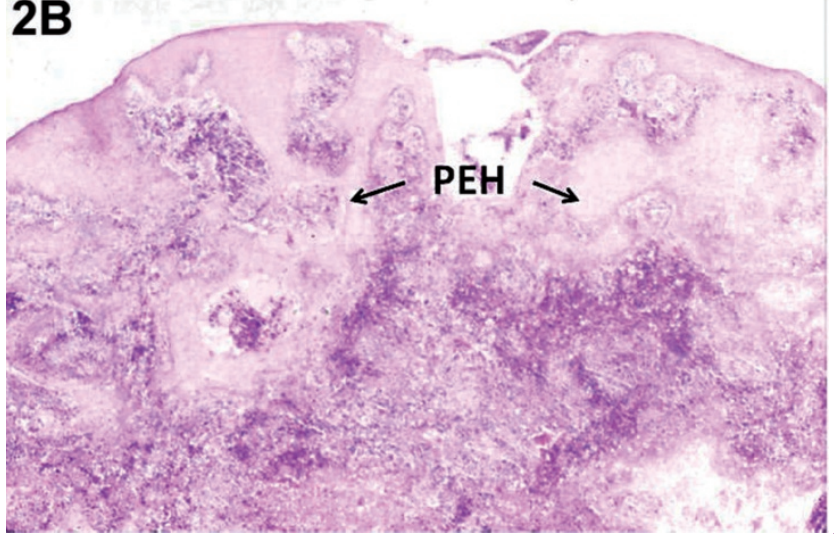

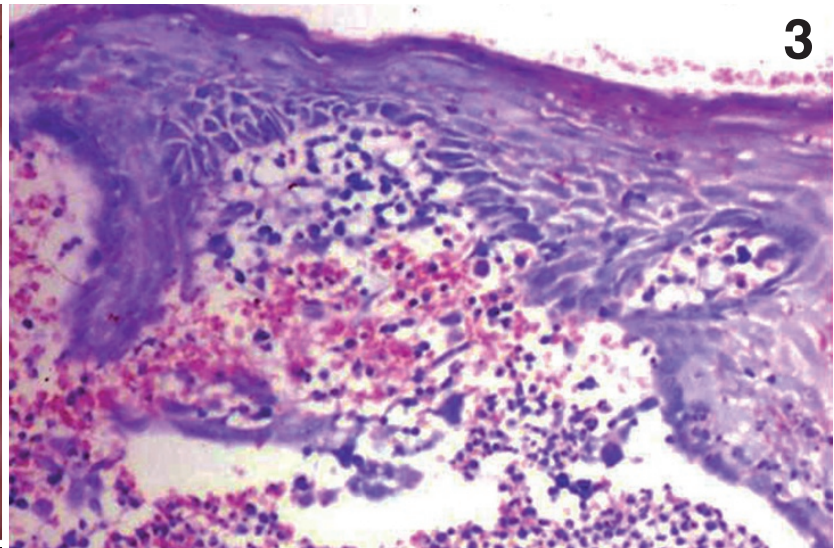

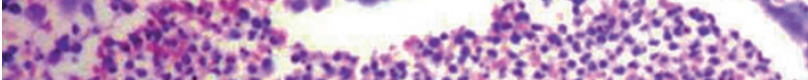

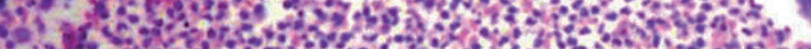
-2l 093 H.

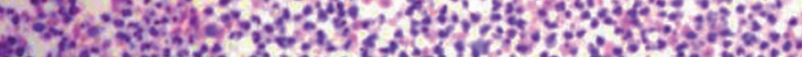
A.

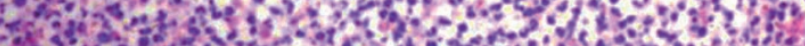

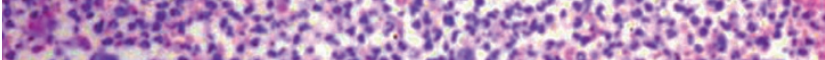

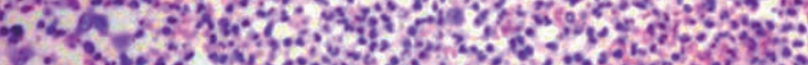

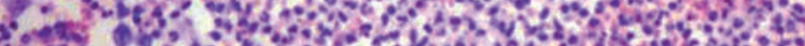

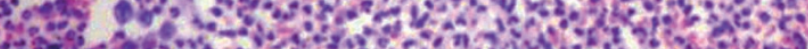

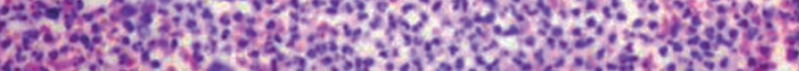

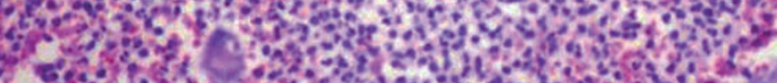

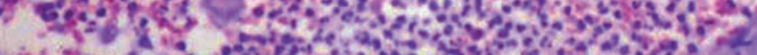

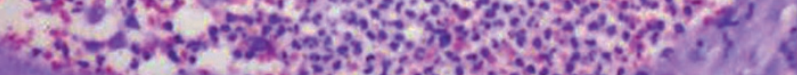

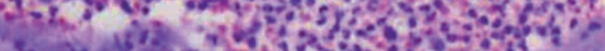

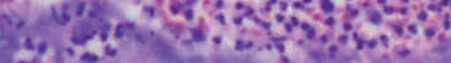
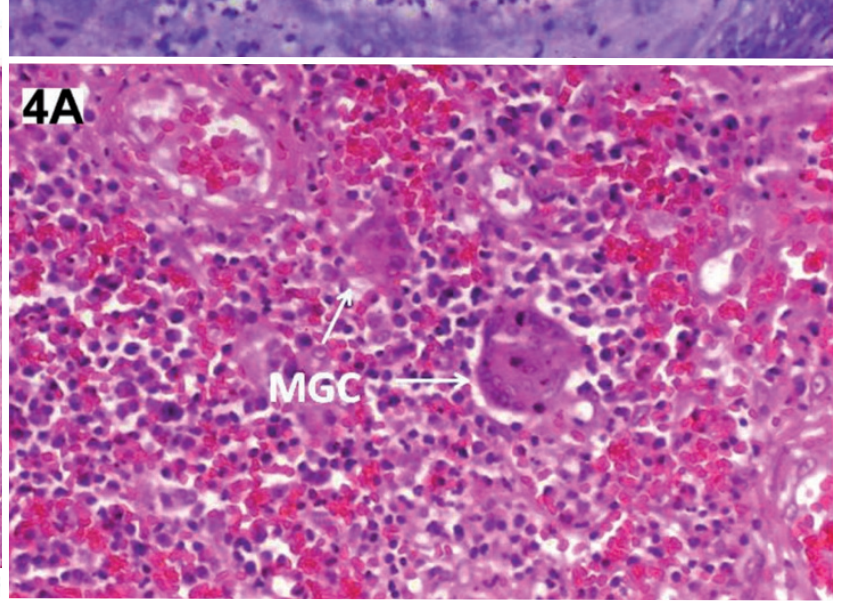

4B

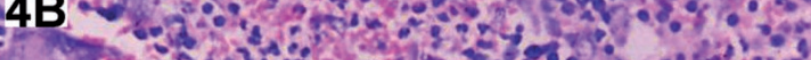

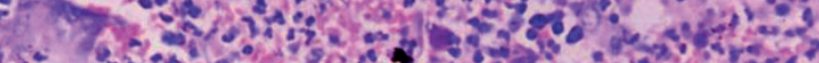

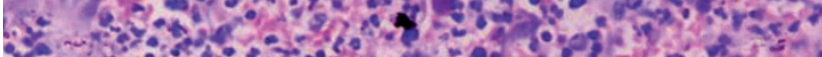

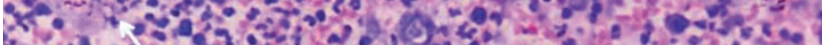

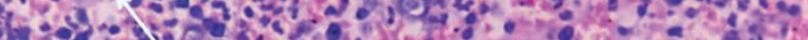

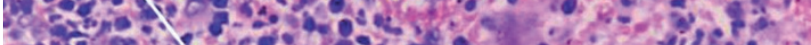

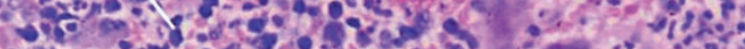

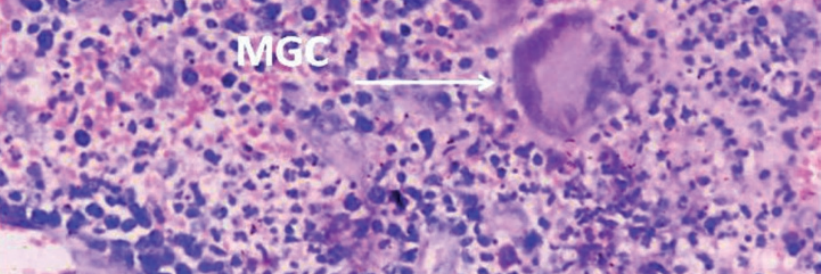



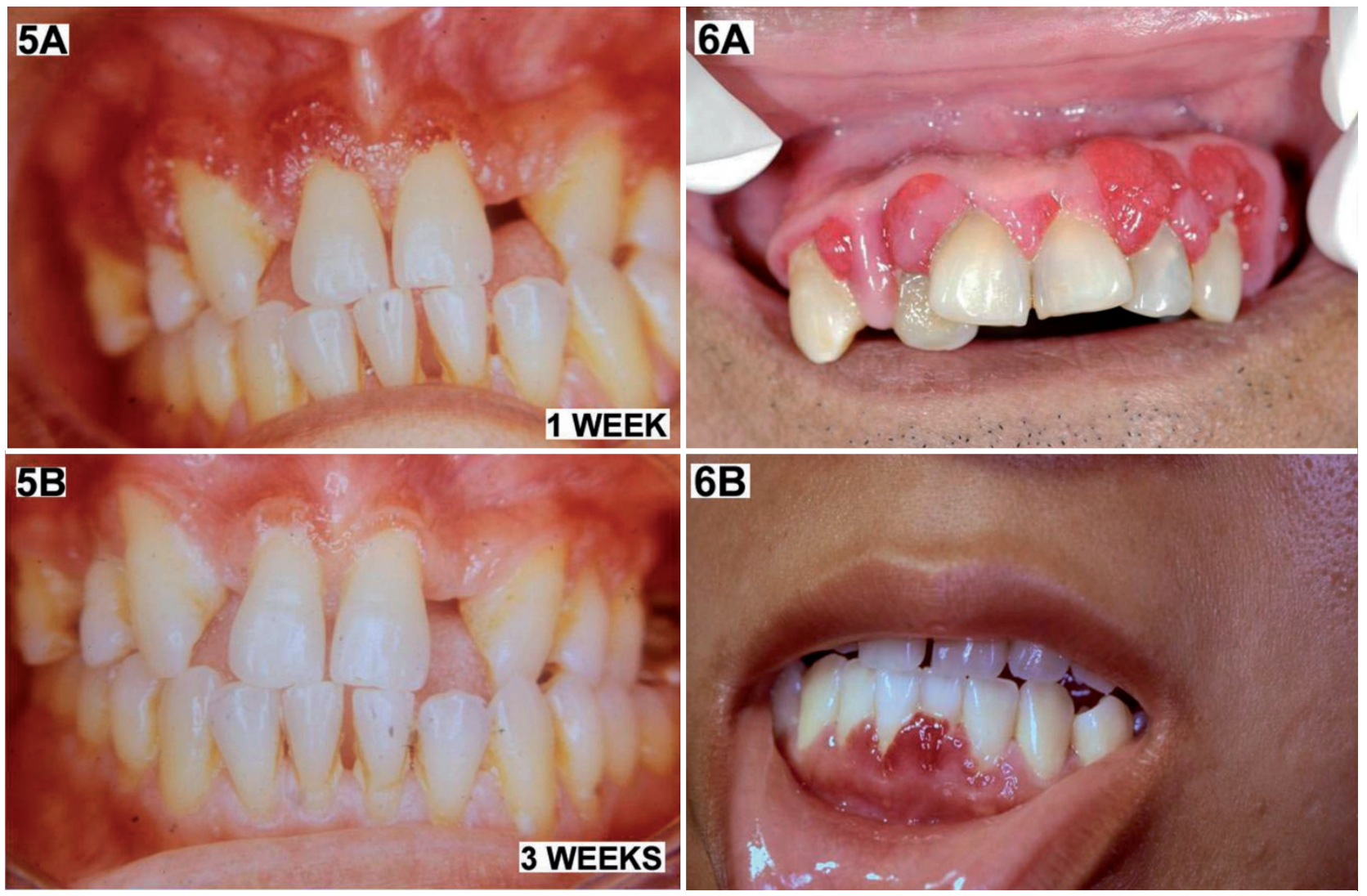

Fig. 5. A-B. Clinical review of the present case shows regression of the gingival lesions at one week (A) and complete resolution at three weeks (B).

Fig. 6. A-B. Examples of other gingival overgrowths that may present as vascular fleshy swellings. A, dilantin-induced hyperplasia and $\mathrm{B}$, pyogenic granulomas.

Microscopic examination of the lesional tissues showed covering parakeratinized stratified squamous epithelium with an irregular lobular surface and exhibiting pseudoepitheliomatous hyperplasia (Fig. 2A$\mathrm{B})$. In the underlying connective tissues, a granulomatous inflammatory response was observed. A diffuse mixed inflammatory cell infiltrate comprising mostly neutrophils which formed subepithelial abscesses (Fig. 3 ) and smaller numbers of eosinophils, plasma cells and lymphocytes was present. Scattered multinucleated giant cells, some resembling Langhan-type giant cells with horse-shoe arrangement of their nuclei, were occasionally seen (Fig. 4A-B). However necrotizing vasculitis was not evident. Special stains were negative for fungi and mycobacterial bacilli. Based on these histological findings and in correlation with the clinical presentation of strawberry gingivitis, a diagnosis of Wegener's granulomatosis was made.

\section{MANAGEMENT}

The patient was referred to the Medical Department for a systemic work-out. Investigations yielded normal indices for haemoglobin, complete blood count, erythrocyte sedimentation rate and urine analysis. Chest $\mathrm{X}$-rays were also normal. ANCA test was not available.

A decision was taken to treat the patient with prednisolone $60 \mathrm{mg}$ daily and cyclophosphamide $100 \mathrm{mg}$ daily.

At one and three weeks post-treatment, a dental evaluation showed that the gingival swellings had completely resolved (Fig. 5A-B). Subsequent reviews were uneventful.

\section{DisCuSSION}

The American College of Rheumatology (ACR) recommended that the diagnosis of Wegener's granulo-

4 Fig. 1. A. Strawberry gingivitis has a characteristic appearance. The present case consists of large lobulated, purplish-red swellings involving the labial gingival mucosa and extending from the distal of the right maxillary first premolar to the distal of the left maxillary central incisor. B. Examination of the palatal mucosa shows no evidence of similar lesions.

4 Fig. 2. A-B. Incisional biopsy specimen of present case shows pseudoepitheliomatous hyperplasia (PEH) (arrows) and a mixed inflammatory infiltrate with abundant neutrophils and eosinophils in the underlying connective tissues (A, H\&E; B, PAS; original magnification $\times 25$ ).

Fig. 3. Subepithelial microabscess formation $(\mathrm{PAS}$; original magnification $\times 100)$.

4 Fig. 4. A-B. Scattered multinucleated giant cells (MGC) (arrows) are present in the lesional connective tissue area, occurring side by side with mixed inflammatory cell infiltrate.(A, H\&E; B, PAS; original magnification $\times 200)$. 
matosis can be made if two of the following criteria are fulfilled:1) ulcerative lesions in oral mucosa or nasal bleeding or inflammation, 2) nodules, fixed infiltrates or cavities in chest radiograph, 3) abnormal urinary sediment and 4) granulomatous inflammation on biopsy [7]. In the present case, criteria 1) and 4) were met. Strawberry gingivitis and demonstration of granulomatous inflammation in the gingival biopsy supported the diagnosis of this case as Wegener's granulomatosis. However, there was no history of epistaxis nor pulmonary infiltrates or abnormal urine findings in our patient. Furthermore, his routine blood analysis was also noncontributory.

In the absence of other systemic findings, other differential diagnoses need to be considered for fleshy vascular gingival growths. Gingival enlargements are not an uncommon finding in the daily clinical practice. Majority of these lesions represent reactive hyperplasias due to plaque-related inflammatory gingival disease [13]. Drug-induced gingival hyperplasia as encountered in patients on dilantin (Fig. 6A), cyclosporine or nifidepine therapy formed the other clinical important group of gingival entities [14]. Vascular lesions that may occur in the gingiva included haemangiomas [15], pyogenic granulomas (Fig. 6B), peripheral giant cell granulomas [13] and the more ominous entities such as Kaposi's sarcoma [16]. Oral potentially malignant disorders may also present with a significant vascular component [17]. Therefore histological verification supported by clinical and other investigations are necessary to differentiate the lesions from strawberry gingivitis.

In summary, a case of strawberry gingivitis presenting as the first sign of Wegener's granulomatosis is described. The clinical lesson from this case report is that early recognition of the characteristic presentation of the gingival lesions of this potentially fatal disorder led to the timely management of the disease.

\section{REFERENCES}

1. Fletcher CDM, Unni K, Mertens K. Pathology and genetics of tumours of soft tissue and bone. World Health Organization, Geneva, 2002

2. Lilly J, Juhlin T, Lew D, Vincent D, Lilly G. Wegener's granulomatosis presenting oral lesions. A case report. Oral Surg Oral Med Oral Pathol 1998; 85: 153-157.

3. Ponniah I, Shaheen A, Shankar KA, Kumaran MG. Wegener's granulomatosis: The current understanding. Oral Surg Oral Med Oral Pathol Oral Radiol Endod 2005; 100:265-270.

4. Bhatt V, Hall TJ. Strawberry gingival enlargement as only manifestation of Wegener's granulomatosis. Br J Oral Maxillofac Surg 2009; 47:500.
5. Lourenco SV, Nico MMS. Strawberry gingivitis: An isolated manifestation of Wegener's granulomatosis. Acta Derm Venereol 2006; 86:90-91.

6. Ruokonen H, Helve T, Arola J, Hietanen J, Lindqvist C, Hogstrom J. "Strawberry gingivitis" being the first sign of Wegener's granulomatosis. Eur J Int Med 2009; 20:651653.

7. Steward C, Cohen D, Bhattacharyya I, Scheitler L, Riley S, Calamia K, Migliorati C, Baughman R, Langford P, Katz J.. Oral manifestations of Wegener's granulomatosis: a report of three cases and a literature review. J Am Dent Assoc 2007; 138:338-348.

8. Cohen RE, Cardoza TT, Drinnan AJ, Aquirre A, Neiders ME. Gingival manifestations of Wegener's granulomatosis. J Periodontol 1990; 61:705-709.

9. Eufinger $\mathrm{H}$, Machtens E, Akuamoa-Boateng E. Oral manifestations of Wegener's granulomatosis. Review of the literature and report of a case. Int J Oral Maxillofac Surg 1992; 21:50-53.

10. Napier SS, Allen JA, Irwin CR, McCluskey DR. 'Strawberry gums' - a case of Wegener's granulomatosis. Br Dent J 1993; 175:327-329.

11. Manchanda Y, Tejasvi T, Handa R, Ramam M. Strawberry gingiva: a distinctive sign in Wegener's granulomatosis. J Am Acad Dermatol 2003; 49:335-337.

12. Shiboski CH, Regezi JA, Sanchez HC, Silverman Jr S. Oral lesions as the first clinical sign of microscopic polyangiitis: a case report. Oral Surg Oral Med Oral Pathol Oral Radiol Endod 2002;94:707-711.

13. Savage NW, Daly CG. Gingival enlargement and localized gingival overgrowths. Aust Dent J 2010; 55: 55-60.

14. Jaiarj N. Drug-induced gingival overgrowths. J Mass Dent Soc 2003; 52: 16-20.

15. Rashmi MS, Alka KD, Seema C. Oral hobnail hemangioma. Quintessence Int 2008; 39:507-510.

16. Lager I, Altini M, Coleman H, Ali H. Oral Kaposi's sarcoma: A clinicopathologic study from South Africa. Oral Surg Oral Med Oral Pathol Oral Radiol Endod 2003; 96: 701-710.

17. Siar $\mathrm{CH}$, Oo VPA, Nagatsuka H, Nakano K, Ng KH, Kawakami T. Angiogenic squamous dysplasia-like phenomenon in oral epithelial precursor lesions. Eur J Med Res 2009;14:315-319.

Received: March 10, 2011 / Accepted: May 25, 2011

Address for correspondence:

Toshiyuki Kawakami, PhD, Professor,

Hard Tissue Pathology Unit,

Matsumoto Dental University Graduate School of Oral Medicine,

1780 Hirooka-Gobara,

Shiojiri, 399-0781 Japan

Phone and Fax: +81-263-51-2035

E-mail: $\quad$ kawakami@po.mdu.ac.jp 